\title{
Endosonographic Appearance of Gastric Adenomyoma
}

\author{
K.-M. Chu \\ Division of Upper Gastrointestinal \\ Surgery, Department of Surgery, \\ University of Hong Kong Medical Center, \\ Queen Mary Hospital, Hong Kong, China
}

Corresponding Author

K.-M. Chu, M.S., F.A.C.S.

Division of Upper

Gastrointestinal Surgery

Department of Surgery

University of Hong Kong Medical Center

Queen Mary Hospital

Pokfulam

Hong Kong

China

Fax: $\quad$ + 852-2819-4221

E-mail: chukm@hkucc.hku.hk

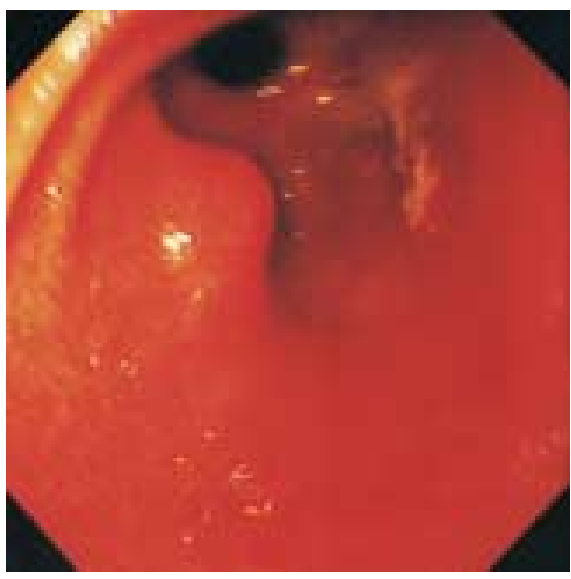

Figure 1 A 37-year-old lady presented with intermittent epigastric pain which she had experienced for 1 year. She was otherwise healthy. The physical examination was unremarkable, and the results of basic laboratory investigations were all within normal limits. An ultrasound scan of the abdomen was normal. Upper endoscopy revealed the presence of a $1.5-\mathrm{cm}$ submucosal nodule in the anterior antrum

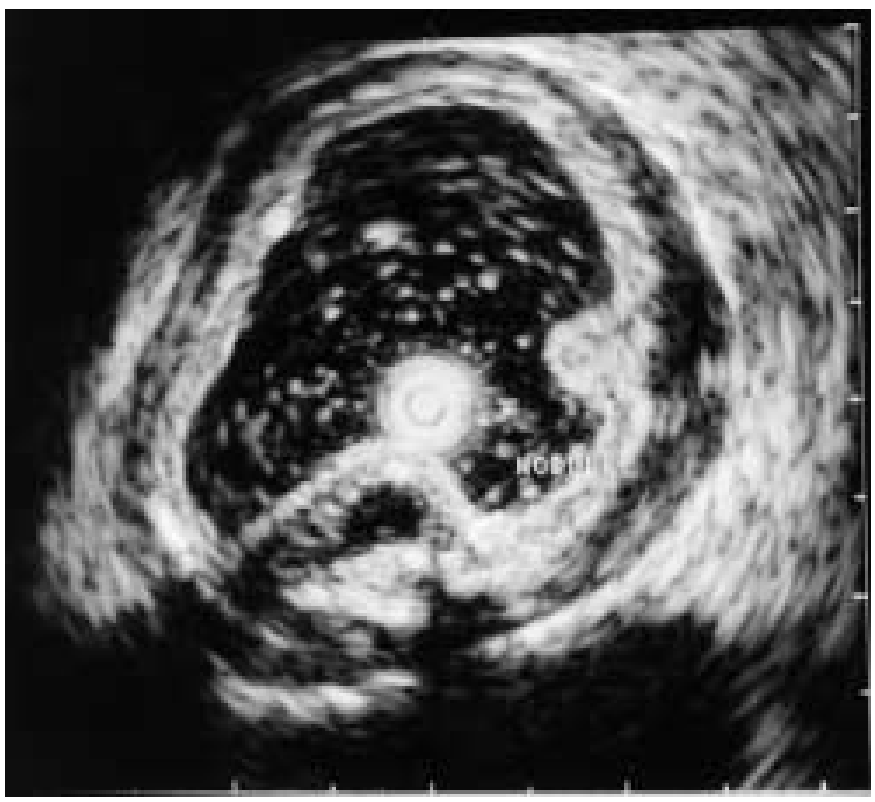

Figure 2 Endoscopic ultrasound (EUS) was performed using the Olympus catheter-probe UM-2R (12 MHz) (Olympus, Tokyo, Japan). The nodule was related to the submucosa of the stomach, with a cystic center lined by a hyperechoic rim. The nodule was resected laparoscopically. Histopathological examination of the resected specimen revealed features of adenomyoma. At the time of writing, the patient has remained asymptomatic for 36 months. The author believes this to be the first description of the EUS appearance of gastric adenomyoma 